\title{
SYZYGIES OF SMALL RANK
}

\author{
BY E. GRAHAM EVANS AND PHILLIP GRIFFITH ${ }^{1}$
}

Let $R$ be a local domain and $M$ a finitely-generated $R$-module. The module $M$ is called a $k$ th syzygy if it sits back $k$ steps in projective resolution of some $R$ module. The syzygy problem asks if nonfree $k$ th syzygies of finite projective dimension necessarily have rank greater than or equal to $k$. We have established the following result.

THEOREM. Let $R$ be a local Cohen-Macaulay domain containing a field and let $M$ be a finitely-generated kth syzygy of finite projective dimension and of rank less than $k$. Then $M$ is free.

For $M$ an $R$-module and $m$ an element of $M$ we define the order ideal, $O_{M}(m)$, to be the ideal which consists of the images of $m$ under homomorphisms of $M$ to $R$. The proof proceeds by considering the height of particular order ideals, $O_{M}(m)$, in relation to the projective dimension of $M$.

Specifically, if $M$ is a $k$ th syzygy of the $R$-module $N$ and if $x_{1}, \ldots, x_{k-1}$ is an $R$ sequence, then $\operatorname{Tor}_{k}^{R}\left(N, R /\left(x_{1}, \ldots, x_{k-1}\right)\right)$ is zero. However, if $O_{M}(m)$ is contained in the ideal $\left(x_{1}, \ldots, x_{k-1}\right)$, then considering the map from $M$ to the free module $F$ which occurs in the resolution of $N$, the image of $m$ is contained in $\left(x_{1}, \ldots, x_{k-1}\right) F$. Thus $\operatorname{Tor}_{k}^{R}\left(N, R /\left(x_{1}, \ldots, x_{k-1}\right)\right)$ is nonzero. However, if $O_{M}(m)$ is contained in the ideal $\left(x_{1}, \ldots, x_{k-1}\right)$, then considering the map from $M$ to the free module $F$ which occurs in the resolution of $N$, the image of $m$ is contained in $\left(x_{1}, \ldots, x_{k-1}\right) F$. Thus $\operatorname{Tor}_{k}^{R}\left(N, R /\left(x_{1}, \ldots, x_{k-1}\right)\right)$ is nonzero. This idea is the key step in Gröbner's proof of Hilbert's syzygy theorem [4]. It shows that the ideal $O_{M}(m)$ tends to have height at least $k$ if $M$ is a $k$ th syzygy. On the other hand the height of $O_{M}(m)$ tends to be bounded by the rank of $M$. While modules of small rank can have elements with the height of $O_{M}(m)$ large, we show that such phenomena cannot occur in the minimal counterexample to the syzygy problem.

The hypothesis that $R$ contains a field is needed to insure the existence of maximal Cohen-Macaulay modules [6] which replace the module $R /\left(x_{1}, \ldots, x_{k-1}\right)$ in our modification of Gröbner's proof [4]. The hypothesis that $R$ is CohenMacaulay is needed to apply the Auslander-Bridger criterion [1] for a module to

Received by the editors December 4, 1980.

1980 Mathematics Subject Classification. Primary 13C10, 13C15, 13D05; Secondary $14 \mathrm{F05}, 14 \mathrm{M} 07,14 \mathrm{M} 10$.

${ }^{1}$ Both authors were partially supported by N.S. F. 
be a $k$ th syzygy. In particular, $M$ is a $k$ th syzygy of finite projective dimension over a Cohen-Macaulay ring $R$ precisely when $M$ is $S_{k}$. We recall that $M$ is $S_{k}$ means that $\operatorname{depth}_{R} M_{P} \geqslant \min (k$, height $P$ ) for all prime ideals $P$ of $R$.

This result has several consequences in commutative algebra and algebraic geometry (cf. [2] for a more complete list). We mention two which seem among the most interesting.

COROLlary 1. Let $R$ be a regular local ring containing a field and let $P$ be a prime ideal of height two such that the module of dualizing differentials, $\Omega_{R / P}^{0}$, is cyclic. Then $R / P$ is a complete intersection. We note that $\Omega_{R / P}^{0}$ is cyclic if $R / P$ is a unique factorization domain.

Corollary 2. Let E be an algebraic vector bundle on $\mathrm{P}^{n}$ of rank $k<n$ which is not a sum of line bundles. Then at least one of the twists of the cohomology groups $H^{1}(E), \ldots, H^{k-1}(E)$ is nonzero.

This corollary is mentioned by Hartshorne [5]. Indeed it is equivalent to the original problem for the case of graded syzygies over rings of polynomials.

The preceding results will appear in our article [3].

\section{REFERENCES}

1. M. Auslander and M. Bridger, Stable module theory, Mem. Amer. Math. Soc. No. 94 (1969).

2. W. Bruns, E. G. Evans and P. Griffith, Syzygies, ideals of height two and vector bundles, J. Algebra 67 (1980), 143-162.

3. E. G. Evans and P. Griffith, The syzygy problem, Ann. of Math. (to appear).

4. W. Gröbner, Algebraische Geometrie. II. Allgemeine Theorie der Kommutativen Ringe und Körper, Bibliographisches Institut, Mannheim, 1970.

5. R. Hartshorne, Algebraic vector bundles on projective spaces: A problem list, Topology 18 (1979), 117-128.

6. M. Hochster, Topics in the homological theory of modules over commutative rings, CBMS Regional Conf. Ser. in Math., no. 24, Amer. Math. Soc., Providence, R. I., 1976.

DEPARTMENT OF MATHEMATICS, UNIVERSITY OF ILLINOIS AT URBANACHAMPAIGN, URBANA, ILLINOIS 61801 